\title{
Out of the darkness
}

\author{
The Black Cloud \\ by Fred Hoyle \\ Heinemann/Harper and Brothers: 1957
}

\section{Jay M. Pasachoff}

From time to time, I remember an image of a dark interstellar cloud invading the Solar System and of scientists wondering what to do. The memory is not real, of course, but conjured up from reading Fred Hoyle's novel The Black Cloud long ago. I was inspired to re-read it by Simon Mitton's recent excellent biography of Hoyle, Fred Hoyle: A Life in Science (Aurum Press, 2005), and found the story to be much more up-to-date than I had expected.

Scientists read science fiction by their peers for the scientific ideas and the characterizations of people they may know, not for the literary style. The science has held up fairly well - interstellar clouds are still important for astrophysics. What I find particularly well done in Hoyle's book is the portrayal of how scientists work, figuring out basic parameters and making first-order estimates based on inadequate data. The reasoning processes of the scientists come up in several contexts, such as how they work out when the cloud will arrive, or that the cloud is slowing down, not speeding up under gravity, as it approaches the Sun.

What I was not expecting was the applicability of political matters in the book to today's situations. In a week when a US federal judge issued an opinion forcing the authorities to release the names of prisoners at Guantanamo Bay that had long been kept secret, the book's portrayal of the US and UK governments' attempt at secrecy over the black cloud's approach seemed particularly relevant. Further, when informed that a dark cloud was approaching, the British home secretary muses: "I am quite sure ... that we can dig up some regulation that will enable us to detain the two of them, the Astronomer Royal and the man from Cambridge." The prime minister responds that "the Statue Book doesn't go back so many centuries for nothing".

A main theme of the book is the conflict between the scientists, pure in thought, and the politicians of both the United States and Britain. At one point the politicians fire nuclear rockets at the cloud, not consulting the scientists (who have contrived to sequester themselves under isolated and extremely comfortable circumstances in a country house in the Cotswolds), thereby risking the future of humanity.

Looking back in terms of today's torrent

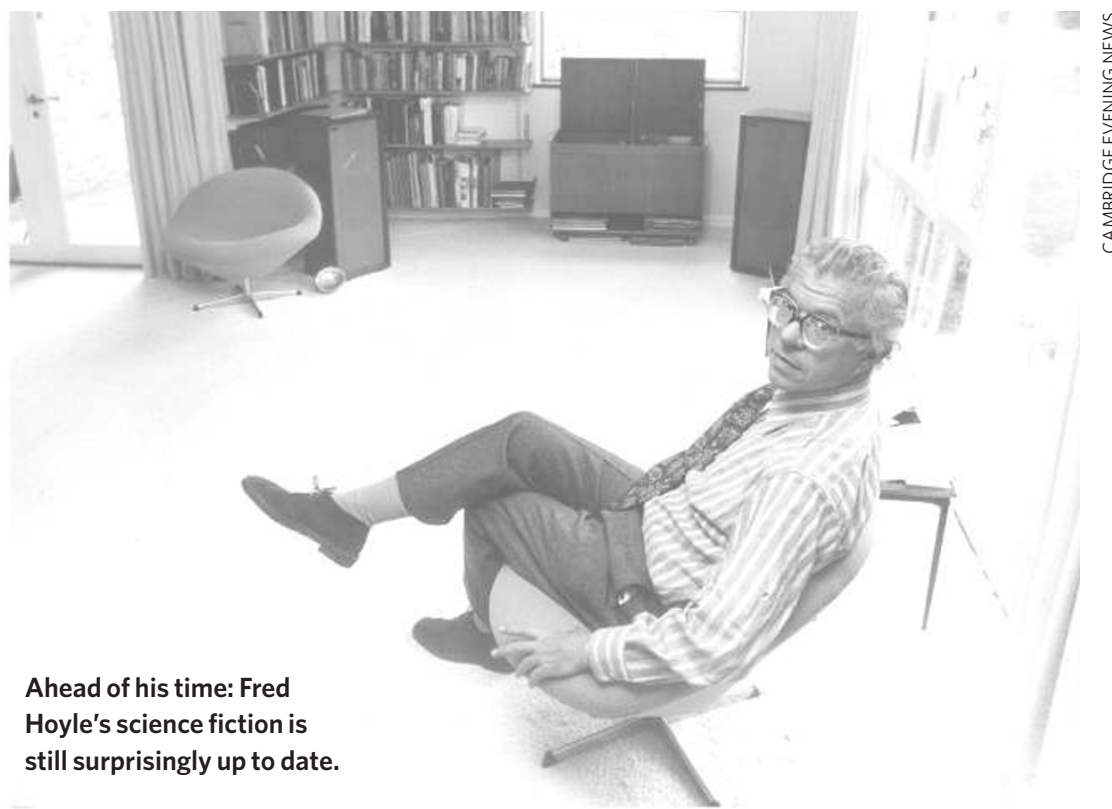

of digital information, it is fun to see Hoyle's speculations from the mid-1950s of information transfer rates, starting with the "human mouth transmits information at some two words per second".

One can imagine the characters as they were then - no doubt with Hoyle as the brilliant, young British scientist and Willy Fowler of Caltech as the Pasadena genius. The astronomer royal of the time has been superseded in my mind by today's Martin Rees, just the brilliant theoretician needed to save the world. The original need for secrecy in the 1957 novel proved prescient for the withholding of information about the discovery of pulsars in 1968 for some weeks, when it was thought that Jocelyn Bell's bits of "scruff" might be signals from little green men. Since then, a protocol has been set up for how scientists should act and release information in case a believable signal is received from one of the projects in the search for extraterrestrial intelligence.

The rivalry among Palomar Observatory scientists, British theoreticians, and British and Australian radioastronomers has certainly evolved, but the basic pros and cons of their cooperation rings true.

Hoyle dwells on the relations between scientists and those in other fields. He was writing at the same time that C. P. Snow was formulating his lectures on 'The Two Cultures and the Scientific Revolution'. Hoyle's scientist may have quoted (actually, slightly misquoted, perhaps on purpose) Shakespeare's "Come kiss me, sweet and twenty/Life's a stuff will not endure" from Twelfth Night but we don't find nonscientists quoting, as Snow demanded, the second law of thermodynamics or its equivalent.

Iconoclastic Hoyle, of course, interspersed a variety of ideas through the book. For example, he pointed out the need for dense urban planning to survive the cold that would be caused when the cloud masked the sun. And I am sure he couldn't resist sticking in some backing for his steady-state theory: "'I would not agree that there ever was a first member', said the Cloud," speaking of its ancestry. "'Oh-ho, there we go. That's one in the eye for the exploding-universe boys'."

Students and the general public could learn some valid statistical ideas from the way the book's scientists analysed the incoming data: "It's no good doing a lot of experiments first and then discovering a lot of correlations afterwards ... Otherwise it's like betting on a race after it's been done." Would that so much pseudoscience and alternative medicine were analysed in this way.

Hoyle may have pushed his speculations - including those about interstellar matter - too far in his later years, but his fertile mind came up with some lasting ideas as well, such as the one about energy levels in the carbon atom that led to our understanding of how most of the chemical elements form. The Black Cloud, on a pleasant but less significant level, remains another testimony to his skills.

Jay M. Pasachoff is Field memorial professor of astronomy, Williams College, Williamstown, Massachusetts 01267, USA. 